Globe

Revue internationale d'études québécoises

"C’est une impossibilité scientifique et matérielle que de garantir l'avenir ". Idiots, aliénés incurables ou déments séniles en congé d'essai, fin $\mathrm{XIX}^{\mathrm{e}}$ début $\mathrm{XX}^{\mathrm{e}}$ siècle "It is Scientifically and Materially Impossible to Predict What May Happen": Trial Leave for the Mentally Retarded, Incurably Insane, and Senile Demented at the Turn of the Twentieth Century

\title{
Marie-Claude Thifault
}

Volume 16, numéro 2, 2013

L’avenir (probable) du passé : le risque et l'histoire du Québec

URI : https://id.erudit.org/iderudit/1025214ar

DOI : https://doi.org/10.7202/1025214ar

Aller au sommaire du numéro

Éditeur(s)

Globe, Revue internationale d'études québécoises

ISSN

1481-5869 (imprimé)

1923-8231 (numérique)

Découvrir la revue

Citer cet article

Thifault, M.-C. (2013). « C'est une impossibilité scientifique et matérielle que de garantir l'avenir ». Idiots, aliénés incurables ou déments séniles en congé d'essai, fin XIX ${ }^{\mathrm{e}}$ début $\mathrm{XX}$ e siècle. Globe, 16(2), 75-94.

https://doi.org/10.7202/1025214ar

\section{Résumé de l'article}

L'Hôpital Saint-Jean-de-Dieu a été, au tournant du $\mathrm{xx}^{\mathrm{e}}$ siècle, le plus grand asile au Canada et par surcroît une référence reconnue internationalement pour l'expertise thérapeutique de ses propriétaires et de ses aliénistes. Le dépouillement de plus de 8000 dossiers médicaux de cette institution psychiatrique a permis de mettre au jour une précieuse correspondance entre les membres de la famille (requérants) des patients et les surintendants médicaux au sujet des congés d'essai. Ces sources de première main révèlent les allées et venues des patients entre l'hôpital et le foyer familial. Elles dévoilent également les appréhensions du futur imputables au retour définitif du patient. Cette étude sur l'histoire culturelle des sensibilités explore les dimensions privée et intime, sous l'angle du " risque ", concernant l'anticipation d'un quelconque malheur générée par la réintégration en milieu familial d'un idiot, d'un aliéné incurable ou d'un dément sénile. Un intérêt particulier est porté sur les discours autour de

" l'événement-non-encore-survenu ", qui motive les requérants à refuser la mise en liberté définitive de leur malade interné à l'hôpital psychiatrique. 
«C’EST UNE IMPOSSIBILITÉ SCIENTIFIQUE ET MATÉRIELLE QUE DE
GARANTIR L'AVENIR ${ }^{1}$. IDIOTS, ALIÉNÉS

\section{INCURABLES OU DÉMENTS SÉNILES EN CONGÉ D'ESSAI, FIN XIX' DÉBUT XX ${ }^{E}$ SIÈCLE²}

\section{MARIE-CLAUDE THIFAULT}

Université d'Ottawa

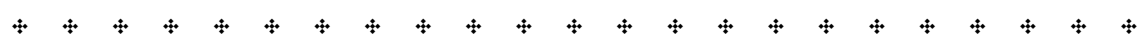

Résumé - L'Hôpital Saint-Jean-de-Dieu a été, au tournant du XXe siècle, le plus grand asile au Canada et par surcroît une référence reconnue internationalement pour l'expertise thérapeutique de ses propriétaires et de ses aliénistes. Le dépouillement de plus de 8000 dossiers médicaux de cette institution psychiatrique a permis de mettre au jour une précieuse correspondance entre les membres de la famille (requérants) des patients et les surintendants médicaux au sujet des congés d'essai. Ces sources de première main révèlent les allées et venues des patients entre l'hôpital et le foyer familial. Elles dévoilent également les appréhensions du futur imputables au retour définitif du patient. Cette étude sur l'histoire culturelle des sensibilités explore les dimensions privée et intime, sous l'angle du "risque», concernant l'anticipation d'un quelconque malheur générée par la réintégration en milieu familial d'un idiot, d'un aliéné incurable ou d'un dément sénile. Un intérêt particulier est porté sur les discours

$$
\div+\div
$$

1. AHL-HL. DM 9292. Lettre du surintendant médical adressée à l'épouse d'un patient, 7 décembre 1909.

2. L'auteure désire remercier les évaluateurs externes pour leurs judicieux commentaires qui ont permis d'améliorer la version préliminaire de cet article. 
autour de "l'événement-non-encore-survenu ", qui motive les requérants à refuser la mise en liberté définitive de leur malade interné à l'hôpital psychiatrique.

\section{$\div \quad \div$ \\ "It Is Scientifically and Materially Impossible To Predict What May Happen": Trial Leave for the Mentally Retarded, Incurably Insane, and Senile Demented at the Turn of the Twentieth Century}

Abstract - At the turn of the $20^{\text {th }}$ century, Saint-Jean-de-Dieu Hospital was the largest asylum in Canada and the therapeutic skills of its operators and psychiatrists were internationally renowned. Consultation of over 8000 medical files from this psychiatric institution uncovered valuable correspondence between the families of patients (petitioners) and medical superintendents on the subject of trial leave. These primary sources describe the comings and goings of patients between the hospital and the family home. They also reveal fears for a future when the patient might return home permanently. This study of the cultural history of sensibilities examines, from the perspective of "risk", the private and intimate dimensions of the contemplation of potential misfortune caused by the return of a family member who was deemed mentally retarded, incurably insane, or senile demented. In particular, the article focuses on the discourse surrounding "events that had yet to occur" that prompted petitioners to reject the unconditional release of a family member from the psychiatric hospital.

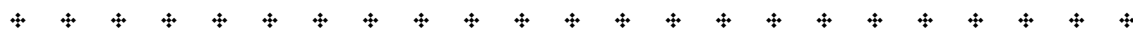

Il a déjà été proposé que l'Hôpital Saint-Jean-de-Dieu a représenté pour des milliers de familles une solution aux problèmes que leur posait la garde de leur " fou $^{3}$ ». Cette "techniqu[e] de contrainte ${ }^{4}$ " mise en œuvre comme stratégie de survie est ici revisitée afin d'approfondir, dans le cadre de la pratique des congés d'essai, les sentiments qui dominent les requérants et motivent ces derniers à refuser la mise en congé définitif de leur malade interné à l'hôpital psychiatrique. En fait, l'idée du retour de «l'idiot, de l'aliéné incurable ou du dément sénile " place tous les membres de la famille dans l'attente anxieuse d'un éventuel drame qui planerait dorénavant au-dessus de leur vie.

$$
\div \div \div
$$

3. Cette idée de l'exploitation stratégique des asiles par les familles a été avancée par André Cellard et Marie-Claude Thifault (André Cellard et Marie-Claude Thifault, "The Uses of Asylums: Resistance, Asylum Propaganda, and Institutionalization Strategies in Turn-of-the-Century Quebec», James E. Moran et David Wright (dir.), Mental Health and Canadian Society: Historical Perspectives, Montréal, McGill-Queen's University Press, 2006, p. 97-116).

4. Michel FouCAulT, "Il faut défendre la société », Dits et écrits II. 1976-1988, Paris, Gallimard, 2001, p. 125 .

5. Selon les appellations utilisées dans le libellé de la Loi sur les Asiles d'aliénés de 1909 (Art. 4152, S.R.Q.). 
Les études sur le sujet de la folie aux $\mathrm{XIX}^{\mathrm{e}}$ et $\mathrm{XX}^{\mathrm{e}}$ siècles, depuis les quinze dernières années, se sont avérées sensibles à l'identité de l'aliéné, à sa provenance et à ses liens sociaux et familiaux. L'historiographie tant américaine, anglaise, canadienne que française tend de plus en plus à montrer que de complexes interactions sociales, hors du contrôle du milieu asilaire, interviennent dans l'internement des aliénés. Quelques auteurs ont tenté de donner la parole aux internés au sein de leur étude ou, pour le moins, de faire comprendre l'organisation de la vie d'un aliéné au sein de l'asile 7 . Ces recherches ont enrichi l'historiographie sur la folie de nouvelles dimensions, telles que le quotidien au sein de l'asile, le rôle de la famille dans le processus d'internement ainsi que les relations entre les malades et leurs gardiens. Entre autres, Geoffrey Reaume s'est intéressé à l'expérience asilaire des patients du Toronto Hospital for the Insane en documentant le quotidien des patients et leur réseau de relations avec leur famille et la communauté ${ }^{8}$. Cet ouvrage donne une voix aux patients et, selon l'auteur, leurs témoignages nous instruisent sur ce que signifie être interné dans un établissement pour malades mentaux. Ces dernières années, nous constatons aussi le développement d'une approche inclusive par laquelle chercheurs et ex-psychiatrisés collaborent au développement d'une histoire de la folie et de la psychiatrisation?

La présente étude sur l'histoire culturelle des sensibilités explore les dimensions privée et intime, sous l'angle du "risque », concernant l'anticipation d'un quelconque malheur générée par la réintégration en milieu familial d'un idiot, d'un aliéné incurable ou d'un dément sénile. Comprenons qu'il

$$
+4 \div
$$

6. Catherine Cox, Negotiating Insanity in the Southeast of Ireland, 1820-1900, Manchester, Manchester University Press, 2012; Laurence GUIGNARD, Hervé GUILLEMAIN et Stéphane TisON (dir.), Expériences de la folie. Criminels, soldats, patients en psychiatrie (XIX'-XXe siècles), Rennes, Presses universitaires de Rennes, 2013; James E. MORAN, Committed to the State Asylum. Insanity and Society in NineteenthCentury Quebec and Ontario, Montréal/Kingston, McGill-Queen's University Press, 2000; Thierry NOOTENS, Fous, prodigues et ivrognes: familles et déviance à Montréal au XIX siècle, Montréal/Kingston, McGill-Queen's University Press, 2007 ; Roy PORTER et David WRIGHT (dir.), The Confinement on the Insane. International Perspectives, 1800-1965, Cambridge, Cambridge University Press, 2003.

7. André CELlaRD et Marie-Claude THIFAUlt, Une toupie sur la tête. Visages de la folie à Saint-Jean-deDieu, Montréal, Boréal, 2007 ; Catharine COLEBORNe, Madness in the Family: Insanity and Institutions in the Australasian Colonial World, 1860-1914, Basingstoke, Macmillan, 2010 ; Patricia D'ANTONIO, Founding Friends. Families, Staff, and Patients at the Friends Asylum in Early Nineteenth-Century Philadelphia, Cranbury, Bethlehem, Lehigh University Press, 2006 ; Patricia E. PRESTWICH, «Reflections on Asylum Archives and the Experience of Mental Illness in Paris ", Journal of the Canadian Historical Association, New Series, vol. 23, nº 2, 2012, p. 324-343.

8. Geoffrey ReAUME, Remembrance of Patients Past, Patient Life at the Toronto Hospital for the Insane, 1870-1940, Don Mills, Oxford University Press, 2000; Akihito SuZUKI, Madness at Home: The Psychiatrist, the Patient, and the Family in England, 1820-1860, Berkeley, University of California Press, 2006.

9. Brenda A. LeFranÇOIS, Robert MenZIES et Geoffrey ReAume (dir.), Mad Matters : A Critical Reader in Canadian Mad Studies, Toronto, Canadian Scholar's Press, 2013; Nérée ST-AMAND et Eugène LEBLANC, Osons imaginer. De la folie à la fierté, Moncton, Our Voice/Notre voix, 2008. 
est ici question de sentiments à l'égard de facteurs de dangerosité plutôt que de "réponse [s] technique $[s]$ aux risques "calculables", et donc assurables de la société industrielle ${ }^{10}$ ». Cela dit, un intérêt particulier se pose sur le discours autour de "l'événement-non-encore-survenu ${ }^{11}$ ", qui motive les requérants à refuser la mise en liberté définitive de leur protégé - du moins, supposément sous leur responsabilité - en congé d'essai. Cette étude participe également à une relecture des pratiques psychiatriques avant la première grande vague de désinstitutionnalisation qu'a connu le Québec dans les années 1960 et à une rectification d'un point d'histoire ${ }^{12}$.

L'ouverture de l'Asile Saint-Jean-de-Dieu en 1873 et la naissance de l'État-providence sont les principaux repères chronologiques sur lesquels repose notre étude. Elle s'inscrit au cours de la période précédant une vaste campagne d'hygiène mentale mise sur pied, à la fin des années 1920, par le Comité d'hygiène mentale de la province de Québec ${ }^{13}$. Un exercice propagandiste, au sens premier du terme, en quête d'une société meilleure qui "met en lumière le rôle de l'État, de l'Église, de la science et de la technique pour légitimer ses propositions et ses avancées sanitaires, sociales et économiques entourant la gestion de la folie ${ }^{14}{ }$. En fait, à l'ère du libéralisme économique, bien que l'État au tournant du XXe siècle contribue partiellement au

$$
\div+\div
$$

10. Martin PetiTClerC, «L'État-providence, la société du risque et les fondements de la communauté politique", David NigeT et Martin PetiTClerC (dir.), Pour une histoire du risque. Québec, France, Belgique, Montréal, Presses universitaires du Québec, 2012, p. 205.

11. Ulrich BECK, La société du risque. Sur la voie d'une autre modernité, Paris, Flammarion, 2001, p. 60.

12. La désinstitutionnalisation en santé mentale, soit le phénomène d'intégration des personnes psychiatrisées au sein de la société, n'a pas retenu l'attention de beaucoup d'historiens québécois jusqu'à présent. Les précurseurs de ce champ d'études au Québec sont notamment les sociologues Françoise Boudreau (Françoise BOUDREAU, De l'asile à la santé mentale. Les soins psychiatriques: histoire et institutions, Montréal, Éditions Saint-Martin, 2003) et Henri Dorvil (Henri DORVIL, "Les caractéristiques du syndrome de la porte tournante à l'Hôpital Louis-H. Lafontaine", Santé mentale au Québec, vol. 12, ${ }^{\circ} 1$, 1987, p. 79-89; et "La désinstitutionnalisation : du fou de village aux fous des villes ", Bulletin d'histoire politique, vol. 10, n 3, 2002, p. 88-104) ainsi que le psychiatre Hubert Wallot (Hubert WaLLOT, Peuton guérir d'un passé asilaire? Survol de l'histoire socio-organisationnelle de l'hôpital Rivière-des-Prairies, Montréal, Publication HMH, 2006; et La danse autour du fou: entre la compassion et l'oubli. Survol de l'histoire organisationnelle de la prise en charge de la folie au Québec depuis les origines jusqu'à nos jours, Beauport, Publications HMH, 1998). Fidèles aux analyses anti-institutionnelles, ces auteurs se sont surtout intéressés au mythe de l'asile "curatif " en définissant l'asile comme lieu de régulation sociale et avant tout comme lieu d'abandon et de déshumanisation. Récemment, Perreault et Thifault ont proposé, dans le cadre du collectif "Après l'asile - After the Asylum » dirigé par Erika Dick dans Histoire sociale/Social History (2011), une interprétation novatrice sur le sujet, dans la mesure où leur démonstration révèle clairement l'existence de pratiques de désinstitutionnalisation dès la première moitié du XXe siècle (MarieClaude Thifault et Isabelle Perreault, "Premières initiatives d'intégration sociale des malades mentaux dans une phase de pré-désinstitutionnalisation : l'exemple de Saint-Jean-de-Dieu, 1910-1950", Histoire sociale/Social History, vol. 44, no 88, 2011, p. 197-222.

13. Robert BASTIEN et Isabelle PERREAUlT, "Propagande d'hygiène mentale au Québec dans les années 1930 ", Lien social et Politiques, vol. 67, 2012, p. 85-105.

14. Ibid., p. 88 . 
financement des asiles d'aliénés, les idiots, les imbéciles et les déments demeurent largement sous la responsabilité de leur famille... si famille il y $\mathrm{a}^{15}$ !

Le dépouillement de plus de 8000 dossiers médicaux parmi ceux conservés dans les archives de l'Hôpital Louis-H.-Lafontaine ${ }^{16}$ a permis de mettre au jour une précieuse correspondance entre les membres de la famille (requérants) des patients et les aliénistes au sujet des congés d'essai - vacances signées par le surintendant médical qui autorise les familles à sortir leur malade pour une période de trois mois. Ces sources de première main révèlent, sur plus d'un demi-siècle, les allers-retours des patients entre l'hôpital et le foyer familial. Elles dévoilent les appréhensions du futur imputables au retour définitif du patient et expliquent, en partie, la situation d'encombrement qu'a connue Saint-Jean-de-Dieu. De plus, elles permettent d'insuffler des données nouvelles en ce qui a trait aux pratiques entourant «l'isolement thérapeutique " et ce mode de gestion de la dangerosité.

Notre corpus de correspondances à l'étude couvre les admissions à Saint-Jean-de-Dieu entre 1873 et 1921. Précisément, tous les dossiers de 1873 à 1898 et, pour le XXe siècle, les dossiers d'une année sur trois de 1900 à 1921 ont été dépouillés. Parmi les 8537 dossiers de notre base de données ${ }^{17}$, seulement ceux dans lesquels étaient colligées des informations sur le congé d'essai ont été sélectionnés pour ensuite, parmi ceux-ci, n'en retenir que 226. Considérant la minceur de la majorité des dossiers, cette collecte de 226 dossiers est somme toute assez impressionnante, puisqu'ils contiennent une correspondance suffisamment explicite sur le sujet des congés d'essai pour documenter notre enquête.

$$
4+4
$$

15. Notez que $70 \%$ des dossiers médicaux entre les années 1873 et 1921 de Saint-Jean-de-Dieu ne contiennent rien d'autre qu'un ou deux formulaires d'admission et, par conséquent, aucune trace du maintien de liens familiaux. À ce sujet, voir: André Cellard et Marie-Claude Thifault, "The The Uses of Asylums: Resistance, Asylum Propaganda, and Institutionalization Strategies in Turn-of-the-Century Quebec Uses of Asylums: Resistance, Asylum Promotion and Institutionalization Strategies ", James E. Moran et David Wright (dir.), Mental Health and Canadian Society: Historical Perspectives, Montréal, McGill-Queen's University Press, 2006, p. 97-116.

16. Les dossiers médicaux de l'Hôpital Saint-Jean-de-Dieu sont conservés dans les archives de l'Hôpital Louis-H.-Lafontaine.

17. Cette base de données a été constituée au cours de notre projet doctoral (Marie-Claude THIFAULT, "L'enfermement asilaire des femmes au Québec: 1873-1921", thèse de doctorat (histoire), Université d'Ottawa, 2003. 
TABLEAU 1

POPULATION ASILAIRE DE SAINT-JEAN-DE-DIEU, SORTIE EN CONGÉ D'ESSAI ${ }^{18}$

\begin{tabular}{|c|c|c|}
\hline ANNÉE & TOTAL DES ADMISSIONS & $\begin{array}{c}\text { \% DE LA POPULATION } \\
\text { EN CONGÉ D'ESSAI }\end{array}$ \\
\hline 1903 & 206 & 27 \\
\hline 1906 & 252 & 29 \\
\hline 1909 & 304 & 32 \\
\hline 1912 & 371 & 28 \\
\hline
\end{tabular}

Bien qu'il s'agisse d'une faible représentation, considérant que $29 \%$ des patients admis au cours des années 1903, 1906, 1909 et 1912 (tableau 1) ont bénéficié d'un ou de plusieurs congés d'essai, nous estimons que les destins asilaires de ces 226 individus sont de précieuses données pour découvrir, à la fin du XIX ${ }^{\mathrm{e}}$ siècle et au début du $\mathrm{XX}^{\mathrm{e}}$ siècle, des pratiques psychiatriques, trop peu connues, en lien avec la collaboration expresse des familles.

Les propos de Robert Castel, tenus lors de la réédition de La Gestion des risques ${ }^{19}$ en 2011, guident notre démarche dans cet univers du "risque" qui ne retient que depuis très récemment l'attention des historiens ${ }^{20}$. Nous avons été particulièrement interpellée par l'idée qu'émet Castel sur la transition entre la naissance des asiles au début du XIX ${ }^{\mathrm{e}}$ siècle et la culture psychologique des années 1970, au cours de laquelle la notion de "risque» prend le pas sur celle de dangerosité " pour appréhender et gérer les catégories de la population qui posent problème ${ }^{21}$ ". Le phénomène a également retenu l'attention de Janice Harvey dans son intéressante étude sur le risque et la pauvreté au XIX ${ }^{\mathrm{e}}$ siècle $^{22}$. Par ailleurs, sous cet angle du "risque», nous voulons revoir le concept d'une prise en charge presque totale des populations aliénées dites dangereuses par la psychiatrie classique tout en évaluant la gestion des risques d'alors, et, par conséquent, l'élargissement du filet des comportements déviants et marginaux qui deviennent des manifestations de folie - qui posent problème - au fur et à mesure que se confirme le $\mathrm{XX}^{\mathrm{e}}$ siècle.

$$
+4
$$

18. Ibid., p. 251.

19. Robert CASTEL, La gestion des risques, Paris, les Éditions de minuit, 2011 [1981].

20. David Niget et Martin PetiTClerC (dir.), Pour une histoire du risque, ...op. cit.

21. Ibid., p. 7.

22. Janice HARVEY, «Le risque et la ville au XIXe siècle. Discours et interventions en matière de pauvreté et de santé dans le Montréal anglo-protestant ", David NigET et Martin PeTiTCLERC (dir.), Pour une histoire du risque, ...op. cit. p. 114. 
Pour ce faire, nous avons divisé cet article en trois parties promettant, premièrement, de prendre la mesure des résistances des aliénistes, quand l'état du malade inspire des craintes sérieuses, à accorder un congé d'essai à leurs patients susceptibles de représenter un danger trop important pour euxmêmes ou leur entourage. Deuxièmement, lorsqu'au contraire l'état du malade donne la plus entière satisfaction et qu'un congé d'essai serait bénéfique pour le malade, nous jaugeons les arguments des aliénistes pour convaincre les familles de reprendre leur malade. Finalement, avant de conclure, nous présentons les principaux obstacles limitant l'action des aliénistes et expliquant plutôt la résistance des familles à voir leur protégé définitivement libéré de l'institution psychiatrique.

\section{QUAND L'ÉTAT DU MALADE INSPIRE DES CRAINTES SÉRIEUSES}

Les questions concernant les sorties des patients internés à Saint-Jean-deDieu, à la fin du XIX ${ }^{\mathrm{e}}$ siècle et au début du XXe siècle, sont sous le contrôle du surintendant médical. Celui-ci est généralement disposé à favoriser les congés de ses patients au sein de leur famille comme le confirment les propos du $\mathrm{D}^{\mathrm{r}}$ Villeneuve devant un certain scepticisme soulevé sur ce sujet : "en règle générale, nous favorisons au contraire la sortie de nos malades en congés d'essai lorsque nous présumons qu'ils puissent en bénéficier ${ }^{23}$ ". Nous sommes également en mesure de confirmer cet état de fait pour l'avoir constaté dans le cadre de différents projets de recherche sur Saint-Jean-de-Dieu, menés en collaboration avec Cellard et Perreault, s'intéressant à l'impact du rôle de la famille sur l'encadrement thérapeutique et la durée de l'internement des patients psychiatriques, spécifiquement, dans l'analyse des correspondances ${ }^{24}$. En ce qui concerne la présente étude, il s'avère que dans $11 \%$ (24) des dossiers de notre échantillon, le surintendant médical est formel et déconseille vigoureusement aux familles de sortir leur malade. Explicitement, parce qu'il est imprudent et même dangereux de laisser certains malades retourner dans

$$
\div+\div
$$

23. Archives Hôpital Louis-H.-Lafontaine (AHL-HL). Dossier médical (DM) 8349. Lettre du surintendant médical adressée au curé de Saint-Gabriel-de-Brandon, 20 novembre 1906. Il est noté que toutes les citations tirées des dossiers médicaux de l'Hôpital Saint-Jean-de-Dieu ont été retranscrites telles que présentées dans leur version originale. Nous n'avons pas cru bon d'ajouter les [sic] après les erreurs considérant qu'elles sont trop nombreuses et que cela alourdirait inutilement les fragments de textes souvent difficiles à lire.

24. Voir entre autres: André CELlard et Marie-Claude THIfAult, Une toupie sur la tête, ...op. cit.; Marie-Claude THIFAULT, "Sentiments et correspondances dans les dossiers des femmes internées à l'Hôpital Saint-Jean-de-Dieu, fin XIX ${ }^{\mathrm{e}}$ siècle, début $\mathrm{XX}^{\mathrm{e}}$ ", Recherches féministes, vol. 21, $\mathrm{n}^{\circ} 2,2008$, p. 127-142 ; et Marie-Claude THIFAulT et Isabelle PERREAUlT, «The Social Integration of the Mentally Ill in Quebec Prior to the Bédard Report of 1962 ", Canadian Bulletin of Medical History/Bulletin canadien d'histoire de la médecine, vol. 29, no 1, 2012, p. 125-150. 
leur famille dans les cas de délire ou encore dans ceux présentant des caractères irritables et impulsifs. Néanmoins, force est de constater que les facteurs de dangerosité exposés pour motiver le refus d'accorder l'autorisation à un requérant de sortir son protégé semblent pour de rares familles être un argument peu persuasif, comme le démontre la correspondance entre le surintendant médical et l'épouse d'un patient dont l'essentiel des échanges est rapporté ici. De fait, l'épouse renouvelle pour la sixième fois, auprès du surintendant médical, une demande de congé d'essai pour son mari atteint d'un délire de persécution et interné pour cette cause depuis 13 mois. L'aliéniste, après avoir présenté l'état physique et mental du malade, ouvre la porte à la possibilité d'un congé conditionnelle à ce que la requérante assume l'entière responsabilité de la sortie du malade.

Je dois vous dire que je ne partage pas du tout votre opinion, au sujet de la sortie, même, sous congé d'essai, de [votre mari], et je suis de plus convaincu, que les chances sont, que son retour dans sa famille occasionnerait de grave inconvénients, sinon des dangers. Sa santé est assez bonne, mais il est encore très délirant, et nous ne pouvons vous offrir absolument aucune garantie. [...] Je ne pourrais vous permettre de le retirer de l'asile sous congé d'essai, que si vous me donniez l'assurance qu'il serait surveillé constamment, jour et nuit, et que vous nous dégageriez de toute responsabilité pour les accidents, soit à lui-même, ou aux autres, qui pourraient se produire après son départ de l'asile ${ }^{25}$.

Trois semaines plus tard, l'épouse demande d'autres nouvelles sur l'état de son mari et cette fois le surintendant réaffirme sa position : "Je ne puis autoriser maintenant la sortie de [votre mari], parce que dans l'état où il se trouve, je considère qu'il ne pourrait que être dangereux, pour lui-même $[\ldots]^{26}$ ". L'impossibilité d'assurer une surveillance constante au malade lors d'un congé d'essai est souvent mentionnée comme étant le principal obstaclerisque - à laisser sortir un patient susceptible d'être dangereux. Selon Castel, «[e]n termes de logique, le diagnostic de dangerosité rabat la catégorie du possible sur celle du réel, sous prétexte que le possible est - plus ou moins probable ${ }^{27}$ ". Les résultats de l'examen du $\mathrm{D}^{\mathrm{r}}$ Grosbois, soumis au $\mathrm{D}^{\mathrm{r}}$ Devlin et confirmant le danger d'accorder un congé d'essai à une patiente violente, vont dans ce sens :

$$
\div+\div
$$

25. AHL-HL. DM 9326. Lettre du surintendant médical adressée à l'épouse du patient, 14 avril 1910. 26. AHL-HL. DM 9326. Lettre du surintendant médical adressée à l'épouse du patient, 6 juin 1910.

27. Robert CASTEL, La gestion des risques..., op. cit., p. 144. 
Les troubles du caractère sont très accusés. Il y a chez [cette malade], irritabilité et variabilité d'humeur, réaction impulsives passagères, colères violentes, etc. etc. Sans provocations elle se précipite sur les autres malades en criant et gesticulant et les sœurs ont été obligées de la mettre dans la Salle Ste.Madeleine, car pendant ses accès délirants cette malade doit être l'objet d'une surveillance constante ${ }^{28}$.

Ainsi, bien que la dangerosité demeure une notion mystérieuse selon Castel, celui-ci reconnaît que, pour la psychiatrie classique, la "dangerosité a été une croix particulièrement lourde à porter, car elle désigne au cœur de son fonctionnement un coefficient incompressible d'arbitraire. Toute "conduite à tenir" devant un sujet supposé dangereux [...] peut être soupçonnée d'être ou bien trop laxiste, ou bien trop répressive ${ }^{29}$ ». Il n’en demeure pas moins qu'il faut considérer que notre corpus à l'étude révèle plutôt une tendance favorable aux congés d'essai, qui se confirme à mesure que le $\mathrm{XX}^{\mathrm{e}}$ siècle se précise ${ }^{30}$. Par conséquent, plus nombreux sont les cas qui nous permettent de témoigner de la transformation des pratiques psychiatriques dès les premières décennies du XX $\mathrm{XX}^{\mathrm{e}}$ siècle, entre autres en ce qui a trait à la volonté des aliénistes à réintégrer leurs patients au sein de la société, une volonté qui sera toutefois confrontée aux appréhensions des familles inquiètes d'un passage à l'acte imprévisible et violent de la part de leur malade mental ${ }^{31}$.

\section{SON ÉTAT NOUS DONNE LA PLUS ENTIÈRE SATISFACTION}

À la suite d'une demande de la famille qui requiert un certificat médical ou tout simplement des nouvelles sur les progrès de leur malade, les aliénistes semblent s'attarder plus longuement sur l'état de ces patients. Du moins, ils le font assez consciencieusement pour observer le patient et faire un examen spécial et du coup constater que l'état du malade s'est sensiblement amélioré si ce n'est que le malade est suffisamment rétabli pour sortir en congé d'essai. Parfois, c'est plutôt conséquemment à la visite d'un parent que le médecin initie l'examen mental du patient visité et collige des renseignements à son sujet auprès du personnel et de son entourage pour conclure, dans les meilleures circonstances, qu'il n'est pas nécessaire de prolonger son internement ${ }^{32}$.

$$
4+4
$$

28. AHL-HL. DM 7918. Lettre de J.B. de Grosbois, médecin interne, adressée au D Devlin, assistant surintendant médical, 10 avril 1906.

29. Robert CASTEL, La gestion des risques..., op. cit., p. 144.

30. Selon les données recueillies pour notre projet doctoral, en 1890 , à peine $1 \%$ des patientes profitent d'un congé d'essai tandis qu'en 1906 elles sont $30 \%$ et $53 \%$ en 1928 (Marie-Claude THIFAULT, "L'enfermement asilaire des femmes au Québec: 1873-1921", op.cit., p. 245-246).

31. Robert CASTEL, La gestion des risques..., op. cit., p. 144-145.

32. AHL-HL. DM 10684. Lettre du surintendant médical adressée à un proche du patient, 26 juin 1912. 
La famille est informée de ce constat et, par conséquent, autorisée à venir chercher son malade au plus tôt. La stratégie mise en place est, dans un premier temps, d'accorder au patient sa sortie de l'asile pour progressivement, dans un second temps, le réintégrer définitivement au sein de sa famille. Lorsque le patient se trouve dans d'excellentes dispositions, une sortie, sous forme de congé d'essai, est proposée avec la possibilité, si un revirement dans l'état du malade se produit, de le ramener à l'asile, et cela sans nouvelles formalités. Le $\mathrm{D}^{\mathrm{r}}$ Devlin soumet une telle proposition au mari d'une de ses patientes, justement, en excellentes dispositions: "Je vous engage donc à profiter de l'époque des Fêtes pour faire cette tentative et de venir chercher votre femme, la semaine prochaine, afin de lui procurer la joie de revoir ses enfants ${ }^{33}$ ».

Toutefois, la réception d'une lettre de l'aliéniste annonçant que l'état mental d'un malade s'est amélioré est parfois accueillie avec plus d'inquiétude que de soulagement, surtout lorsque cette nouvelle atteste également que le patient est suffisamment rétabli pour quitter l'hôpital. Cette situation oblige, dans certains cas, une épouse ou un époux à envisager de reprendre la vie commune. La décision, par exemple, de retirer son mari de l'asile s'accompagne inévitablement d'appréhension. Les inquiétudes concernant une rechute ou la difficulté à simplement croire à une guérison complète peuvent rendre assez difficile la décision d'aller chercher son malade. C'est sur cette toile de fond qu'un discours sur le «risque» émane des correspondances consultées dans les dossiers médicaux des patients admis à l'Hôpital Saint-Jean-de-Dieu entre 1873 et 1921.

Certes, les hésitations sont nombreuses et la solution pour les apaiser réside dans l'obtention d'un certificat signé du surintendant médical garantissant que le malade est "guéri » et qu'il peut quitter l'asile sans aucun danger de rechute. Une assurance que, malheureusement, aucun aliéniste ne peut donner, car, selon de $\mathrm{D}^{\mathrm{r}}$ Villeneuve, «c'est une impossibilité scientifique et matérielle que de garantir l'avenir ${ }^{34} »$. Bien qu'un état mental satisfaisant ne donne aucune garantie, le congé d'essai demeure, néanmoins, une tentative nécessaire pour justement évaluer, sur une période de trois mois, la lucidité du patient tout en conservant la possibilité de retourner le malade à l'asile sans devoir faire remplir à nouveau les formulaires d'admission. Vraisemblablement insuffisante dans bien des cas, cette garantie place les requé-

$$
\div+\div
$$

33. AHL-HL. DM 8451. Lettre de l'assistant surintendant médical adressée à l'époux de la patiente, 14 décembre 1909.

34. AHL-HL. DM 9292. Lettre du surintendant médical adressée à l'épouse d'un patient, 7 décembre 1909 . 
rants dans une position de défense qui les pousse à refuser de se porter garants de leur malade hors des murs asilaires.

Tapies dans leur retranchement, certaines familles refuseront de recevoir chez eux leur malade, évitant ainsi de prendre le moindre risque. Cette situation s'apparente à celle d'un nommé François, interné le 9 juillet 1919 à l'âge de 69 ans pour aliénation mentale, qui, selon l'avis du $\mathrm{D}^{\mathrm{r}}$ Noël, était apte à sortir en congé d'essai le 20 octobre de la même année. Malheureusement, seule la mort, survenue le 9 juin 1922, le libéra de l'asile. Ni ses trois enfants, ni sa femme, ni ses trois frères n'ont voulu se porter garants de cet homme. C'est le curé de la paroisse qui se chargea de transmettre les doléances de la famille.

La famille, malheureusement, n'est pas capable de recevoir le père. Ils demeurent dans un bloc à suites ${ }^{35}$, très à l'étroit, et tous leurs voisins se rappellent les agissements insensés du pauvre vieux, alors qu'il était ici, et le voyant revenir, les mêmes voisins ne consentiront pas à garder le pauvre vieux dans ce bloc, et il faudra que la famille de [cet homme] se cherche un logis, chose à peu près impossible à trouver dans le moment. De plus, le pauvre vieux leur a fait tant d'ennuis dans le passé, qu'il leur répugnerait énormément de le voir revenir, de crainte que ces mêmes ennuis ne se renouvelassent de nouveau. Veuillez [...] faire tout en votre pouvoir pour qu'on garde le vieux à l'Hôpital St-Jean de Dieu. Bien que pauvres, ils préfèreraient contribuer quelque chose pour sa pension, si c'est nécessaire afin qu'on le garde là ${ }^{36}$.

Effectivement, la décision de recevoir un parent en congé d'essai ne repose pas uniquement sur les membres de la famille puisque le retour du malade peut également être anticipé par le voisinage. Le curé de la paroisse de StMichel-de-Napierville, en 1905, avait quant à lui écrit aux autorités de SaintJean-de-Dieu pour permettre le retour d'une paroissienne "à la condition empressée que les parents en [aient] soin et qu'ils l'empêch[ent] de venir nous troubler dans le village, et surtout à l'église ${ }^{37}$ ", une assurance que le surintendant médical ne peut absolument pas garantir, mais qui demeure une demande récurrente de la part des requérants: "I hope Doctor that she will not be sent back until you are satisfied that she will not cause any more trouble ${ }^{38}$ ".

$$
4+4
$$

35. Mots soulignés dans le document original.

36. AHL-HL. DM 14369. Lettre de l'abbé Jubinville adressée à Monsieur Trépanier, 7 décembre 1909. 37. AHL-HL. DM 7992. Lettre du curé de la paroisse de Saint-Michel-de-Napierville adressée au surintendant médical, 15 décembre 1905.

38. AHL-HL. DM 7445. Lettre du médecin de famille adressée au surintendant médical, 10 octobre 1903. 
La procédure des congés d'essai est étroitement liée aux facteurs économiques non négligeables dans les pressions exercées pour sortir certains aliénés de l'asile. La Loi sur les asiles d'aliénés de 1909 (Art. 4152, S.R.Q.) donne l'autorité nécessaire au secrétaire de la province ou au surintendant médical d'ordonner que les cas chroniques soient envoyés dans leur famille à condition qu'ils ne soient pas une cause de scandale ou de danger.

Le secrétaire de la province ou le surintendant médical sur l'autorisation écrite du secrétaire de la province peut ordonner que les idiots, les aliénés incurables ou les déments séniles sortent de l'asile où ils se trouvent pour être envoyés dans leurs familles, ou chez les personnes tenues en loi à leur entretien, ou dans les hôpitaux dans lesquels on reçoit les vieillards et les malades; pourvu toujours que les malades ainsi libérés ne soient pas une cause de scandale ou de danger ${ }^{39}$.

L'anticipation d'un quelconque malheur générée par la réintégration en milieu familial d'un idiot, d'un aliéné incurable ou d'un dément sénile - selon les appellations utilisées dans le libellé de la loi - devient toutefois un sérieux handicap aux tentatives de sortir cette classe de malades de l'établissement asilaire. Les raisons qui motivent les requérants à refuser la mise en congé d'essai de leur malade interné à l'hôpital psychiatrique reposent en grande majorité sur les expériences difficiles vécues avec le patient avant l'internement et l'idée que son retour place tous les membres de la famille dans l'attente anxieuse d'un éventuel drame qui planerait dorénavant au-dessus de leur vie. Bien que le surintendant médical exprime une grande confiance dans le succès d'un congé d'essai, il n'a cependant d'autres choix que d'être honnête quant aux nombreux facteurs pronostiques défavorables. En effet, il doit s'assurer que le requérant est au fait de l'état physique et mental de son protégé. Voici un extrait plus explicite sur ce sujet:

Il est probable qu'il [le patient] ne recouvrira jamais sa force comme autrefois et qu'il se sentira toujours des effets de cette paralysie. Quant à son état mental, il est assez satisfaisant, car il est calme, docile et ne présente aucune délire. Il est facile de voir cependant, que son esprit est quelque peu affaibli, et qu'il ne pourra jamais recouvrer son activité, comme auparavant. Je serais disposé à le laisser sortir si quelqu' un voulait bien se charger de lui, car il n'est pas encore capable de gagner sa vie et je doute fort qu'il puisse jamais le faire d'une façon effective ${ }^{40}$.

$$
4+4
$$

39. Extrait de l'article de loi tiré d'une lettre du surintendant médical adressée le 20 octobre 1925 à l'épouse du patient. AHLHL, dossier 6518.

40. AHL-HL. DM 9578. Lettre du surintendant médical adressée au neveu du patient, 19 mai 1910. 
Malgré les tentatives des aliénistes de rassurer les requérants, la sérieuse situation d'encombrement qui prévaut à Saint-Jean-de-Dieu, à mesure que se confirme le $\mathrm{XX}^{\mathrm{e}}$ siècle, oblige le surintendant médical à recourir de plus en plus souvent à l'article 4153 de la Loi sur les asiles d'aliénés de 1909 qui stipule que:

Toute personne qui a signé la demande d'internement d'un patient dans un asile, ou toute personne tenue à son entretien en vertu de la loi, doit aller y chercher ce patient, à ses frais, dès qu'elle en est requise par le surintendant médical de tel asile ou par le secrétaire de la Province, sous peine d'une amende de trente piastres ${ }^{41}$.

Néanmoins, considérant la situation d'encombrement que vivent les patients à Saint-Jean-de-Dieu jusque dans les années 1960, nous comprenons que l'article 4153 n'a pas permis d'imposer aux requérants la responsabilité qui leur incombe à retirer leur malade de l'asile. Force est donc de constater les faiblesses de cet article de loi en comparaison à l'argument de poids que sont les sentiments d'inquiétude, de peur ou de désarroi des parents en ce qui concerne "l'événement-non-encore-survenu qui motive l'action ${ }^{42}$ " des requérants à refuser le retour définitif de leur malade interné à l'hôpital psychiatrique.

\section{L'INQUIÉTUDE D'UN ÉVENTUEL MALHEUR}

Les résistances d'une famille à garder leur malade en congé d'essai sont fondées sur ce que Beck qualifie d'«un futur qu'il s'agit d'empêcher d'advenir. [...] C'est l'avenir qui vient s'y substituer, et c'est alors quelque chose d'inexistant, de construit, de fictif qui devient la "cause" de l'expérience et de l'action présentes ${ }^{43}$ ». C'est-à-dire, dans le cas qui nous préoccupe, le refus de consentir à libérer définitivement ledit aliéné, afin d'éviter un éventuel malheur, comme le mentionne la mère d'une petite idiote: «ce qui m'inquiète le plus docteur s'il fallait par malheur qu'elle aurait encore des grandes crises comme auparavant... ${ }^{44}$ ». Parmi tous les dossiers à l'étude, plus de $40 \%$ (94) témoignent de la volonté des parents à prolonger le congé d'essai plutôt qu’à demander la libération définitive de leur malade et cela même après plus de six ou même neuf mois d'extension au congé d'essai initial de trois mois,

$$
4+4
$$

41. AHL-HL. DM 10809. Lettre du surintendant médical adressée au beau-frère de la patiente, 14 novembre 1912.

42. Ulrich BECK, La société du risque..., op. cit., p. 60.

43. Ibid., p. 60-61.

44. AHL-HL. DM 9544. Lettre de la mère de la patiente adressée au surintendant médical, 16 juin 1910. 
comme en témoigne cet extrait : "Je dois vous dire que mon mari ne semble pas avoir encore l'esprit bien lucide, pour plus de certitude si vous vouliez avoir la bonté de m'accorder encore trois mois de congé vous m'obligeriez beaucoup ${ }^{45}$ ". Cette permission expresse de la famille laisse poindre à l'horizon un seul et même désir, celui de ne surtout pas rompre le lien avec l'institution asilaire, afin d'assurer à son pensionnaire, en cas d'urgence, un accès rapide à Saint-Jean-de-Dieu. Considérant que le surintendant médical n'est pas en mesure de confirmer que le spectre de la folie ne reviendra pas hanter les jours de ses malades, le prolongement du congé d'essai s'avère l'unique solution pour convaincre les requérants de reprendre leur malade... du moins temporairement : "Il y a tout lieu de croire que dans quelques temps il nous faudra la faire réinterner ; c'est pourquoi nous vous prions de ne point signer sa sortie définitive ${ }^{46} »$. Une autre lettre exprime les mêmes appréhensions : "[d]epuis quelle est sortie de lasile elle réside avec ses parents et on ma dit quelle nétait pas encore completement gurie. A chaque mois elle éprouve encore de faible attaques de folie. J'espère que vous allez macorder encore quelque mois de congé parce je pense quelle va devenir encore folle avant longtemps ${ }^{47}$ ». Les requérants profitent de cette zone d'ombre, en ce qui concerne l'incertitude quant au pronostic, pour exploiter au maximum le système asilaire promettant de garder ses portes ouvertes aux patients en congé d'essai. De toute évidence, il s'agit d'une mesure dont on peut abuser et que l'on peut craindre, puisque c'est bien lors de telles circonstances, selon Foucault, que "les relations d'assujettissement peuvent fabriquer des sujets ${ }^{48}$ ". Une mesure qui d'ailleurs semble suffisamment abusive pour susciter l'intervention du sous-secrétaire de la province: "Je suis chargé par le Secrétaire de la Province d'attirer votre attention sur la réadmission de Mde [...] sur votre rapport du 22 au 31 août dernier. Dans nos livres il appert que cette personne a [été] déchargée de l'Asile St. Jean-de-Dieu, le 26 Décembre, $1904^{49}$ ». C'est avec célérité que le $\mathrm{D}^{\mathrm{r}}$ Villeneuve répond à cette lettre en informant le secrétaire de la province que la patiente n'a pas été déchargée définitivement de l'hôpital, mais qu'elle a plutôt bénéficié d'un congé d'essai qui a été renouvelé à la demande des parents de trois mois en trois mois "de manière à permettre

$$
+4 \div
$$

45. AHL-HL. DM 7052. Lettre de l'épouse du patient adressée au surintendant médical, 27 septembre 1903.

46. AHL-HL. DM 9294. Lettre de la sœur supérieure de l'Institut des sourdes-muettes adressée au surintendant médical, 28 février 1910.

47. AHL-HL. DM 7302. Lettre de l'époux de la patiente adressée au surintendant médical, 21 septembre 1903.

48. Michel Foucault, «Il faut défendre la société», ...op. cit., p. 124.

49. AHL-HL. DM 4015. Lettre de Jos. Boivin adressée au $D^{r}$ Villeneuve, 27 septembre 1906. 
un réinternement immédiat au cas où la condition de la [malade] aurait exigé cette mesure ${ }^{50}$ ». Les parents ont pu garder cette malade pendant 20 mois avant de la retourner sous les soins du $\mathrm{D}^{\mathrm{r}}$ Villeneuve.

De fait, cette procédure, développée à Saint-Jean-de-Dieu au tournant du $X_{X}{ }^{e}$ siècle et qui s'affirme davantage à partir de 1909, illustre le seul moyen préventif disponible à une époque au cours de laquelle les aliénistes n’ont que l'isolement thérapeutique comme moyen d'assurer à la société sa sécurité. Le congé d'essai pratiqué à Saint-Jean-de-Dieu permet dans les faits de réintégrer les patients dans leur milieu de vie familial tout en assurant une action préventive, celle-ci étant la possibilité de réadmettre le malade avant même qu'un malheur irréparable ne survienne. Plusieurs lettres sollicitant une prolongation du congé d'essai expriment de fortes hésitations à assumer la pleine responsabilité à l'égard de leur protégé et cela même si chez celui-ci aucun signe inquiétant n'est révélé dans ses actions ni dans son comportement. C'est ce que révèle l'extrait de la lettre qui suit, rédigé par le père d'une patiente.

J'ai l'honneur de vous informer que l'état mental de ma petite fille Aurore a été assez bon depuis son départ de l'asile, mais de crainte qu'elle redevienne encore malade dans le cours du printemps, auriezvous la bonté de m'accorder une extension de temps encore pour trois mois pour vous la renvoyer sans certificats si la chose devenait nécessaire ${ }^{51}$.

Nombreuses sont les raisons susceptibles d'être à l'origine d'une nouvelle crise. Selon le médecin de famille d'une patiente en congé d'essai dans sa famille depuis six mois, la belle saison pourrait être critique pour sa patiente: "j'ai vu dernièrement madame [patiente] qui est très bien et elle a toujours été bien depuis son retour. Cependant comme elle était bien il y a un an, ce printemps et les chaleurs de l'été lui ont été défavorables. Je désirerais obtenir une extension du congé, afin de voir comment elle va passer les chaleurs de cet été ${ }^{52} »$. Pour d'autres, il faut s'en remettre à Dieu :

Je dois vous dire que depuis que j'ai voulu aller la ramener à St de Jean de dieu, au 24 mai son état est beaucoup amélioré. Je ne sais si je dois attribuer ce changement suite à la peur qu'elle eut d'être dans

$$
4 \div 4
$$

50. AHL-HL. DM 4015. Réponse du $D^{r}$ Villeneuve adressée au secrétaire de la province, 29 septembre 1906.

51. AHL-HL. DM 7438. Lettre du père de la patiente adressée au surintendant médical, 18 avril 1904.

52. AHL-HL. DM 7466. Lettre du médecin de famille adressée au surintendant médical, 14 mai 1904.

53. AHL-HL. DM 8189. Lettre de la sour de la patiente adressée au surintendant médical, 28 juillet 1908. 
l'obligation de s'en retourner, il s'est produit une grande amélioration dans sa santé. Il est impossible pour moi de dire qu'elle est complètement guérie, ah non, car ces maladies là il faut attendre avec patience l'heure convenue à Dieu de la parfaite guérison mais je puis vous dire sans hésiter quelle est beaucoup et beaucoup mieux, mais pour cela je ne veux pas que vous signiez sa sortie définitive car l'on ne connait pas les secrets de Dieu et qui sait peut-être que plus tard nous aurons besoin encore de vos généreux soins à son égard ${ }^{53}$.

Plusieurs missives adressées aux autorités asilaires, bien qu'elles rapportent que le malade est rétabli et n'expriment aucune explication particulière justifiant la nécessité de prolonger la sortie, ont néanmoins pour principal objet le renouvellement du congé d'essai. En voici deux exemples, le premier daté de 1898: "J'ai le plaisir de vous apprendre que la santé de ma femme est bonne et quelle est presque parfaitement rétablie. Aussi en même temps je vous demande d'être assez bon de renouveler le permis que vous lui avez accordé le 8 septembre dernier de retournée si elle venait à être obliger ${ }^{54}$ ». Le second est daté de 1908: "J'ai à croire à une guérison mais si vous voulez m'accorder une extansion de conger ${ }^{55}$ ». L'auteur de la courte lettre du premier exemple s'assure de conserver l'autorisation légale de retourner sa femme à l'asile lorsqu'il en jugera nécessaire et cela avant même que le premier congé d'essai de trois mois de son épouse arrive à échéance. Le congé d'essai en tant que mesure préventive a le défaut de sévèrement encadrer des gens qualifiés de "population à risque ", même si les facteurs de risque, dans certains cas, sont de nature très abstraite ${ }^{56}$. Nous ne pouvons, par conséquent, faire fi de toute la subjectivité qui accompagne le pronostic de la famille et son pouvoir de confiner à l'enfermement un parent trop peu utile à la cellule familiale, comme le laisse sous-entendre la note suivante : « [j] e vous écri quel que mots pour vous parler de mon mari il tombe encore comme les premiaire foi et pui a la position que ge suis la ge suis pas capable de le gardé bien lontemps la première petite ferdaine qu'il va me faire ma va bien aitre oubliger de lenvoyer mener $"$ " ${ }^{57}$. Bien entendu, les comportements jugés problématiques ne peuvent être dissociés des normes sociales de l'époque, même si aujourd'hui ils seraient tout simplement considérés comme farfelus,

$$
\div+
$$

54. AHL-HL. DM 6881. Lettre de l'époux de la patiente adressée au surintendant médical, 2 novembre 1902.

55. AHL-HL. DM 14215. Lettre de l'époux de la patiente adressée au surintendant médical, 9 mars 1915.

56. Robert CASTEL, La gestion des risques..., op. cit., p. 8.

57. AHL-HL. DM 6554. Lettre de l'épouse du patient adressée au surintendant médical, 15 janvier 1901. 
comme ceux évoqués ici: «je reviens pour renouveler encore son congé de trois autres mois pour maman elle est encore pareille elle sacre elle parle seule elle ne va pas l'église ${ }^{58}$ ". Plus précisément, comme l'a bien documenté Cellard, " [a]u cours des siècles, certains comportements de la folie ont pu attirer l'attention au gré de l'évolution des sensibilités collectives et susciter le rire, la désapprobation ou l'inquiétude. [...] déroger à la pratique religieuse deviendra bientôt un symptôme de folie ${ }^{59}$ ».

Malgré l'assurance d'une extension du congé d'essai et le statut privilégié qu'octroie cette procédure au patient, pour certains, le retour dudit aliéné à l'asile devient inéluctable. La peur d'un danger imminent est la principale raison évoquée dans les missives adressées au surintendant médical, comme dans celle-ci : "Je crois que je vais être dans la pénible obligation de le retourner [mon fils] sous vos soins avant même que le temps de son congé expire car avec mes autres jeunes enfants il est dangereux ${ }^{60} »$. Du moins, c'est le cas dans $15 \%$ (33) des dossiers consultés. Ceux-ci présentent des faits explicites sur ce qui motive les requérants à retourner leur malade à l'hôpital psychiatrique avant la fin du congé. L'état mental «amélioré » du patient mis en congé soulève de sérieux doutes. C'est précisément en cette circonstance qu'apparait dans les dossiers une correspondance entre le requérant ou son porte-parole et le surintendant médical. Ces lettres révèlent définitivement l'appréhension du futur en gardant plus longuement le malade au sein de la famille. Selon le $\mathrm{D}^{\mathrm{r}}$ Manseau, nonobstant la conduite du malade à l'asile, cela n'est pas une preuve d'équilibre mental permettant de croire à un retour au foyer sans soucis pour les parents. Effectivement, depuis l'arrivée à la maison du patient Joseph, bien qu'à ce jour on n'ait rapporté aucune menace de mort, « [le patient] reste mur pour l'asile - que c'est un aliéné dangereux ${ }^{61}$ !». En ces mots, le $\mathrm{D}^{\mathrm{r}}$ Manseau prévient le $\mathrm{D}^{\mathrm{r}}$ Villeneuve de l'urgence de reprendre son malade avant que l'inéluctable ne se produise. Crainte qu'a également soumise, dix ans plus tôt, le trésorier de Hull au $\mathrm{D}^{\mathrm{r}}$ Villeneuve, au nom de la famille d'une jeune patiente en congé dans sa municipalité :

Il y a deux mois passé que vous nous avez renvoyé dans Hull une Dame $[\ldots]$ que nous avions fait interner à l'asyle pour cause de folie,

$$
4+4
$$

58. AHL-HL. DM 6472. Lettre de la fille de la patiente adressée au surintendant médical, 21 septembre 1905.

59. André CelLARD, "L'hippocampéléphantocamelos et autres monstres ordinaires de l'aliénation mentale au Québec", Sylvie FRIGON (dir.), Corps suspect, corps déviant, Montréal, Éditions du remueménage, 2012, p. 121.

60. AHL-HL. DM 8192 Lettre du la mère du patient adressée au surintendant médical, 14 avril 1904.

61. AHL-HL. DM 8920. Lettre du médecin de famille adressée au surintendant médical, 14 août 1908. 
et depuis qu'elle est de retour ici elle n'a cessée d'être folle comme avant son départ et même plus, et nous nous trouvons dans la pénible nécessité de d'adresser à vous de nouveau pour l'admettre à l'asyle et comme c'était un essaie que vous désiriez faire, nous voudrions savoir s'il nous sera nécessaire de renouveler les papier d'examen etc pour obtenir son admission espérant que vous vous empressiez de répondre, vu que la personne semble être une cause de danger pour la famille $[\ldots .$.$] qui l'a en charge { }^{62}$.

À l'idée d'exposer toute la famille à un éventuel danger, la décision de retourner la patiente à l'asile survient parfois assez tôt au début du congé d'essai de trois mois. Par exemple, une mère de famille de Saint-André-Avellin retourne à l'asile après seulement neuf jours passés parmi les siens. Selon les dires de son mari, transmis par le médecin du village, son épouse doit être sous surveillance constante, "elle est devenue dangereuse pour ses voisins, et ses jeunes enfants au nombre de neuf. Elle cherche à s'empoisonner, à étrangler ses enfants, enfin elle est mure pour l'asile ${ }^{63}$ ". Dans un autre cas, celui d'un nommé Prospère, le surintendant médical affirme que la démence sénile de son patient est maintenant plus facile à conduire, puisque ses facultés et ses forces ont graduellement baissé : ses dispositions sont favorables pour tenter une sortie en congé d'essai. Malgré les hésitations de la famille, le vieil homme est sorti, mais revient à l'asile 12 jours après l'obtention de son congé ${ }^{64}$. Dans ce cas, comme dans l'exemple précédent, l'espoir d'heureux changements s'évanouit rapidement pour laisser place à une difficile situation critique à laquelle le requérant refuse de se voir exposer... probablement las d'attendre un signe de guérison pouvant le rassurer. C'est ce qu'exprime la fille d'une patiente apparemment épuisée et découragée de veiller sur sa mère :

[d] epuis son arriver auqu'un changement mental ne s'est opérer en elle, au contraire. Elle devient de plus en plus trouble pour prouver sa folies ge vous cite ces faits suivant elle ne peut sortir seul sans attaquer tous le monde de la rue elle détruit tous le linges soit en le brulant ou bien en le déchirant elle subit des crises toutes les semaines, enfin nous sommes à bout de forces nous ne pouvons plus la garder, nous avons du trouble... ${ }^{65}$

$$
\div
$$

62. AHL-HL. DM 5870. Lettre du trésorier de la ville de Hull adressée au surintendant médical, 15 janvier 1898.

63. AHL-HL. DM 6712. Lettre du $\mathrm{D}^{\mathrm{r}}$ Brossoit adressée au surintendant médical, $1^{\mathrm{er}}$ avril 1901.

64. AHL-HL. DM 6708. Lettre du surintendant médical adressée au médecin de famille, mars 1903.

65. AHL-HL. DM 6770. Lettre de la fille de la patiente adressée au surintendant médical, 11 février 1904. 
Les résistances des familles à reprendre leur malade avec elles sont telles que les aliénistes doivent resserrer leur pratique de "négociation " pour ainsi exercer plus de pression sur les requérants en évoquant la responsabilité qui leur incombe à l'égard du patient interné à Saint-Jean-de-Dieu. Cela se confirme au cours des années 1910 et de la décennie suivante. L'aliéniste n’hésite plus à user des pouvoirs qui lui sont conférés et signe de plus en plus de lettres de la nature suivante: « [j]'ai l'honneur de porter à votre connaissance que l'état mentale de [nom du patient] s'est amendé, et j'ai cru de mon devoir de ne plus le garder dans cet hôpital ${ }^{66}{ }^{\prime}$. Ces missives restées lettres mortes obligent les aliénistes, en dernière instance, à envisager le transfert des idiots, des aliénés incurables et des déments séniles vers des établissements spécialisés dans le soin des malades incurables ${ }^{67}$.

\section{CONCLUSION}

C'est à travers le langage du risque, dans l'exemple des pratiques de congés d'essai à l'Hôpital Saint-Jean-de-Dieu, que nous avons tenté, à l'instar de Hannem et Bruckert, d'illustrer tant l'expérience du stigmate que l'anticipation à être stigmatisé. Cela, selon une perspective individuelle plutôt qu'institutionnelle parce que le "stigma is not merely conceptual, theoretical, and analytic in nature. It is deeply personal, and the language of stigma provides an expressive vehicle to speak of dynamic "everyday" experience "68 ". C'est à partir de la correspondance retracée dans les dossiers médicaux entre les autorités de l'asile et les requérants qu'il nous a été possible de découvrir l'univers privé et intime des familles pour ainsi mieux estimer l'impact «individuel » du stigmate de la dangerosité.

En somme, cette incursion dans l'univers du risque, pour mieux comprendre les sentiments suscités par la perspective d'un congé d'essai et plus spécifiquement celle du retour définitif du malade psychiatrique en milieu familial, a permis de s'intéresser spécifiquement à l'attente anxieuse d'un éventuel drame qui planerait dorénavant au-dessus de la vie de ces familles. Bien qu'abstraite, subjective, voire abusive, cette appréhension de l'avenir de la part des requérants met en lumière des sentiments de peur, d'impuissance, d'épuisement et de honte qui motivent davantage le désir d'internement que

$$
\div+\div
$$

66. AHL-HL. DM 12031. Lettre du surintendant médical adressée à la famille du patient, 21 janvier 1925.

67. AHL-HL. DM 6160. Lettre du surintendant médical adressée à la famille du patient, 17 juillet 1931. 68. Stacey Hannem et Chris BruCKerT (dir.), Stigma Revisited. Implications of the Mark, Ottawa, Presses de l'Université d'Ottawa, 2012, p. 1. 
de libération du malade psychiatrique. Cela dit, nous avons été à même de jauger le poids du stigmate social à l'égard des idiots, des aliénés incurables ou déments séniles, "sujets" d'assujettissement. Force est d'admettre que "l'isolement thérapeutique", comme mode de gestion de la dangerosité, à l'ère de la psychiatrie classique, s'est transformé, faute de garanties suffisantes témoignant d'une complète guérison du malade, en «technique de contrainte» exploitée par les familles. 